\title{
Open-Bite Treatment of a Case by means of Zygomatic Plate Anchorage
}

\author{
Melih Motro, Banu Çakırer
}

\begin{abstract}
Treatment of anterior open bite cases is usually a challenging issue and the treatment results are often hard to maintain. Many treatment modalities were presented throughout the years in the literature where in most, malocclusion is corrected primarily by extrusion of the incisors or preventing passive eruption of posterior teeth. Surgical correction is another main approach in treatment of skeletal openbite cases. However, patients may hesitate to have surgery due to the risks, complexity and costs of this treatment. In this case report a minimally invasive method is shown in which posterior teeth are intruded by using zygomatic anchorage. For this purpose multi purpose implants were placed on the zygomatic buttress area to act as anchorage units. The intrusive force application was started by attaching two $9 \mathrm{~mm}$ NiTi coil springs bilaterally to the bended "I" shaped processes that access to the oral cavity through the mucosa. Posterior dentoalveolar intrusion was achieved in 6 months and the intrusion was maintained with wire ligation between the implant and the molar tubes throughout the treatment. Lower face height to total face height ratio decreased by $1 \%$. $1 \mathrm{~mm}$ overbite was achieved. Normal smile line concomitant with the lower lip contour and esthetic gingival exposure was also achieved. If intrusion of the posterior teeth is necessary skeletal anchorage through zygomatic multipurpose implants can be used as a good treatment alternative to orthognathic surgery.
\end{abstract}

Keywords: Zygomatic Anchorage, Open-bite, Molar Intrusion

\section{Introduction}

Treatment of anterior open bite cases is usually a challenging issue and the treatment results are often hard to maintain. The morphologic pattern in anterior open bite is characterized by increased facial height, increased height of the posterior dentoalveolar structure to compansate the short vertical dimension of ramus and steep

Department of Orthodontics, Faculty of Dentistry, University of Marmara, Istanbul, Türkiye

Corresponding Author:

Melih Motro

Marmara University Dentistry Faculty Department of Orthodontics.

Buyukciftlik Sk. No: 6 Nisantasi-Istanbul, Türkiye

Tel : +905426417794

e-mail : mmotro@gmail.com mandibular plane $(1,2)$. Many treatment modalities were presented throughout the years in the literature where in most, malocclusion is corrected primarily by extrusion of the incisors or preventing passive eruption of posterior teeth (3-5). Surgical correction is another main approach in treatment of skeletal openbite cases. However, patients may hesitate to have surgery due to the risks, complexity and costs of this treatment (6).

In this case report a case treated with a minimal invasive method which was introduced by Erverdi et al. is going to be presented $(7,8)$.

\section{Case Report}

\section{Diagnosis and Treatment Objectives:}

A 26 years old female patient who was referred to the Orthodontics department of Marmara University Dentistry Faculty had a chief complaint of anterior openbite. Clinical examination revealed a Class I dental relationship on the left and Class II on the right side. She had $5 \mathrm{~mm}$ of anterior openbite and $3 \mathrm{~mm}$ overjet. The upper and lower arches were well aligned. She had her upper 1st molar teeth extracted. Therefore, the treatment plan was: 1- to mesialize the upper $2^{\text {nd }}$ left molar teeth into the 1 st molar's space and to wait for the upper left $3^{\text {rd }}$ molar to erupt. 2- to expand the maxilla by means of Surgically assisted Rapid Maxillary Expansion and to intrude the posterior teeth by using the multipurpose implants applied on the zygomatic buttress area (Figure 1).

\section{Treatment Progress:}

After completion of the mesialization with fixed appliances, posterior braces were removed and a modified bonded Hyrax was placed. To achieve a skeletal maxillary expansion,Le Fort I corticotomy was performed and simultanously two MultiPurpose Implants (Tasarım Med, Istanbul, Turkey) were placed on the right and left Zygomatic Buttress areas. After the removal of sutures at the $7^{\text {th }}$ day following the surgery, RME was started and the appliance was activated twice a day for 15 days. Following the completion of expansion, the intrusive force application was started by attaching two $9 \mathrm{~mm} \mathrm{NiTi}$ coil springs (Masel, Bristol, Pa) bilaterally to the bended "I" shaped processes that access to the oral cavity through the mucosa (Figure 2). Posterior dentoalveolar intrusion was achieved in 6 months and the intrusion was maintained with wire ligation between the implant and the molar tubes throughout the treatment. 
Figure 1. Pre-treatment extraoral and intraoral photos
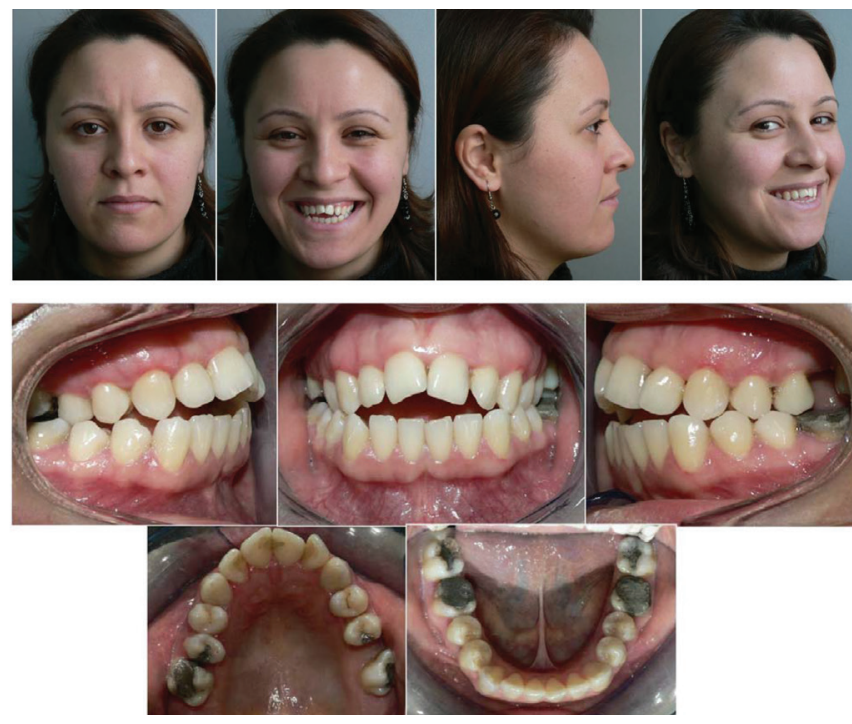

Figure 2. NiTi Coil springs attached to the Zygomatic MultiPurpose implants applying intrusive force to the posterior teeth

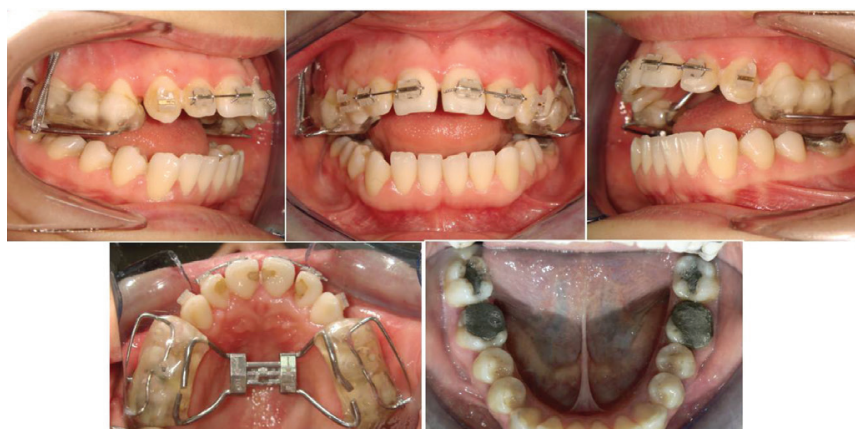

Figure 3. Post-treatment extraoral and intraoral photos
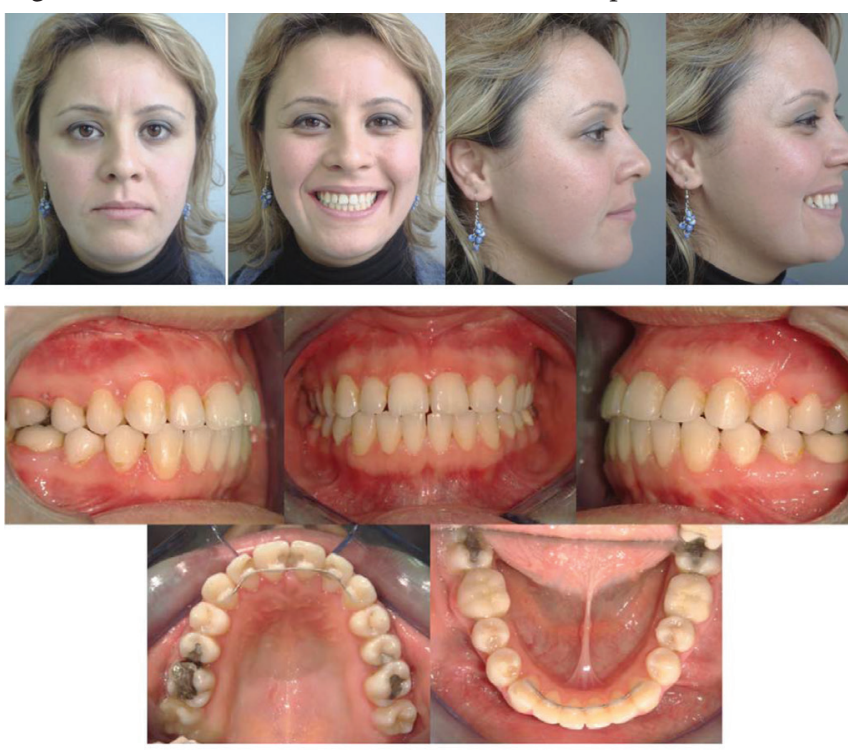

\section{Results}

At the end of treatment Class I canine and molar relationship and anterior open bite correction were achieved with the intrusion of maxillary posterior dentoalveolar segment, eruption and uprighting of the upper incisors. Lower face height to total face height ratio decreased by $1 \%$. $1 \mathrm{~mm}$ overbite was achieved. Normal smile line concomitant with the lower lip contour and esthetic gingival exposure was also achieved (Figure 3).

\section{Discussion}

Multipurpose implants placed on the zygomatic buttress area were shown to be very stable and succesfull anchorage units to intrude posterior teeth. $(7,8)$. Once placed, this anchorage unit can also be used for segmental tooth movement or anchorage reinforcement. Therefore, it is advised to be kept in place till the end of the treatment.

To be able to expand maxilla skeletally in adult patients surgical assistance is necessary. In case of this specific patient it was thought that cortical osteotomies may have accelerated the intrusion movement of the posterior teeth by increasing the circulation and bone remodeling in that area. Having a hyrax screw prevents uncontrolled buccal tipping of posterior teeth.

Patient acceptance of this treatment modality as an alternative to the conventional Le Fort I surgery is better and postoperative pain and discomfort is less. Patient compliance is minimally required (no need for headgear or anterior box elastics etc.) where a good hygiene is necessary. However, some tissue irritation on the cheek mucosa has been monitored because of the arms extending buccally through the acrylic cap of the RME appliance and the "I" processes of the Zygomatic implants.

\section{Conclusion}

If intrusion of the posterior teeth is necessary skeletal anchorage through zygomatic multipurpose implants can be used as a good treatment alternative to orthognathic surgery.

\section{References}

1. Proffit W, Fields H. Contemporary Orthodontics. 2nd ed. St Louis: Mosby, Inc. 1993:128-446.

2. Sassouni V. A classification of skeletal facial types. Am J Orthod. 1969:109-123.

3. Nielsen I. Vertical malocclusions: etiology, development, diagnosis and some aspects of treatment. Angle Orthod. 1991;61:247-260.

4. Küçükkeleş N, Acar A, Demirkaya A A, Evrenol B, Enacar A. Cephalometric evaluation of open bite treatment with NiTi arch wires and anterior elastics. Am J Orthod Dentofac Orthop 1999;116 : 555562 .

5. Kim Y. Anterior openbite and its treatment with multiloop edgewise archwire. Angle Orthod. 1987;57:171-178.

6. Lawry D, Heggie A, Crawfrod E, Ruljancich M. A Review of the managment of anterior open bite malocclusion. Aust Orthod J. 1990;11:147-160

7. Erverdi N, Keles A, Nanda R. The use of skeletal anchorage in open bite treatment: a cephalometric evaluation. Angle orthod. 2004;74(3).

8. Erverdi N, Usumez S, Solak A. New generation open-bite treatment with zygomatic anchorage. Angle Orthod. 2006;76(3):519-526. 